\title{
Composite Fermion Approach to the Quantum Hall Hierarchy: When it Works and Why
}

\author{
Arkadiusz Wójs* and John J. Quinn \\ The University of Tennessee, Knoxville, Tennessee, 37996
}

\begin{abstract}
The mean field composite Fermion (MFCF) picture has been qualitatively successful when applied to electrons (or holes) in the lowest Landau level. Because the energy scales associated with Coulomb interactions and with Chern-Simons gauge field interactions are different, there is no rigorous justification of the qualitative success of the MFCF picture. Here we show that what the MFCF picture does is to select from all the allowed angular momentum $(L)$ multiplets of $N$ electrons on a sphere, a subset with smaller values of $L$. For this subset, the coefficients of fractional parentage for pair states with small relative angular momentum $R$ (and therefore large repulsion) either vanish or they are small. This set of states forms the lowest energy sector of the spectrum.

71.10.Pm, 73.20.Dx, 73.40.Hm
\end{abstract}

When applied to electrons (or holes) in the lowest Landau level, the mean field composite Fermion (MFCF) picture [1] gives a good qualitative description of the low lying states of fractional quantum Hall systems [2]. The original conjecture that the $\mathrm{CF}$ transformation converts a system of strongly interacting electrons into one of weakly interacting composite Fermions cannot possibly be correct because the Chern-Simons interactions among fluctuations are measured on an energy scale (proportional to magnetic field $B$ ) which can be much larger than the energy scale of the Coulomb interactions (proportional to $B^{1 / 2}$ ). Because so many results, both of large numerical calculations and of experiments, can be interpreted in terms of composite Fermions, it is extremely important to understand why the MFCF picture works. This is the problem we address in this letter.

For $N$ electrons on a Haldane sphere [3] (containing at the center a magnetic monopole of charge $2 S h c / e$ ), the single particle states fall into angular momentum shells with $l_{n}=S+n, n=0,1, \ldots$ The $n$th shell is $2 l_{n}+1$ fold degenerate. The CF transformation attaches to each electron a flux tube of strength $2 p_{0}$ flux quanta oriented opposite to the original magnetic field. If the added flux is treated in a mean field approximation, the resulting effective magnetic field seen by one $\mathrm{CF}$ is $B^{*}=B-2 p_{0}(h c / e) n_{s}\left(n_{s}\right.$ is the number of electrons per unit area). An effective CF filling factor $\nu_{0}^{*}$ $\left(\nu_{0}^{*-1}=\nu_{0}^{-1}-2 p_{0}\right)$ and an effective monopole strength $2 S^{*}\left(2 S^{*}=2 S-2 p_{0}(N-1)\right)$ seen by one CF can also be defined. $\left|S^{*}\right|$ plays the role of the angular momentum of the lowest CF shell [4. States belonging to the Jain sequence occur when $\nu_{0}^{*}$ is an integer. For such integral CF fillings, the ground state is a Laughlin [5] incompressible liquid state with angular momentum $L=0$. If $\nu_{0}^{*}$ is not an integer, a partially occupied CF shell will contain $n_{\mathrm{QE}}$ quasielectrons (or $n_{\mathrm{QH}}$ quasiholes). In the MFCF picture these states form a degenerate band of angular momentum multiplets with energy $n_{\mathrm{QE}} \varepsilon_{\mathrm{QE}}$ where $\varepsilon_{\mathrm{QE}}$ is the energy of a single quasielectron (or $n_{\mathrm{QH}} \varepsilon_{\mathrm{QH}}$ for quasiholes). The degeneracy results from the neglect of $\mathrm{QP}-\mathrm{QP}$ interactions in the MFCF approximation [6].

Hierarchy states [7] outside the Jain sequence are obtained (when $\nu_{0}^{*}$ is not equal to an integer) by reapplying the $\mathrm{CF}$ transformation to residual quasiparticles in the partially filled CF shell. In comparing the predictions of the CF hierarchy picture with numerical results for states containing three or four quasiparticles, it is found that the MF approximation is often qualitatively incorrect. Before worrying about the reapplication of the MFCF approach to residual quasiparticles in a partially filled $\mathrm{CF}$ shell, it is very useful to ask why the MFCF picture applied directly to electrons (or holes) in a partially filled shell gives qualitatively correct results. In light of the different energy scales describing Coulomb interactions and Chern-Simons gauge field interactions, the justification cannot lie in a cancellation between these interactions.

We begin by considering the simple case of three electrons in the lowest angular momentum shell, with values of $2 S$ ranging from 2 to 14 . In Tab. I we give the number of times an $L$-multiplet occurs for a given value of $2 S$ (upper table is for even values of $2 S$ and lower one for odd values). Note that the set of values of $L$ at $2 S-2(N-1)=2 S-4$ is always a subset of the set at $2 S$. The validity of this result can be easily established numerically for arbitrary values of $N$ and $2 S$ (including values of $N$ larger than $4\left(S-p_{0}(N-1)+1\right)$, where more than one CF shell is filled). In Fig. 1 the pseudopotential coefficient $V(R)$ is plotted for $2 S=15,20$, and 25 , as a function of $R$, the relative angular momentum of a pair of electrons. $R$ is defined as [3, 6] $L^{\mathrm{MAX}}-L$, where $L^{\mathrm{MAX}}$ is the maximum possible angular momentum of a pair of electrons each with angular momentum $l$, and $L$ is the total angular momentum of the pair. $R$ takes on even values less than or equal to $2 l-1 . V(R)$ is a monotonically decreasing function of $R$ (for the lowest angular momentum shell), and $V(0)$ is considerably larger that $V(2)$. In low-lying energy states, electrons have to avoid having $R=0$ because of the large repulsion. 
TABLE I. The number of times an $L$-multiplet appears for a system of three electrons of angular momentum $l=S$. Top: even values of $2 S$; bottom: odd values of $2 S$. Blank spaces are equivalent to zeros

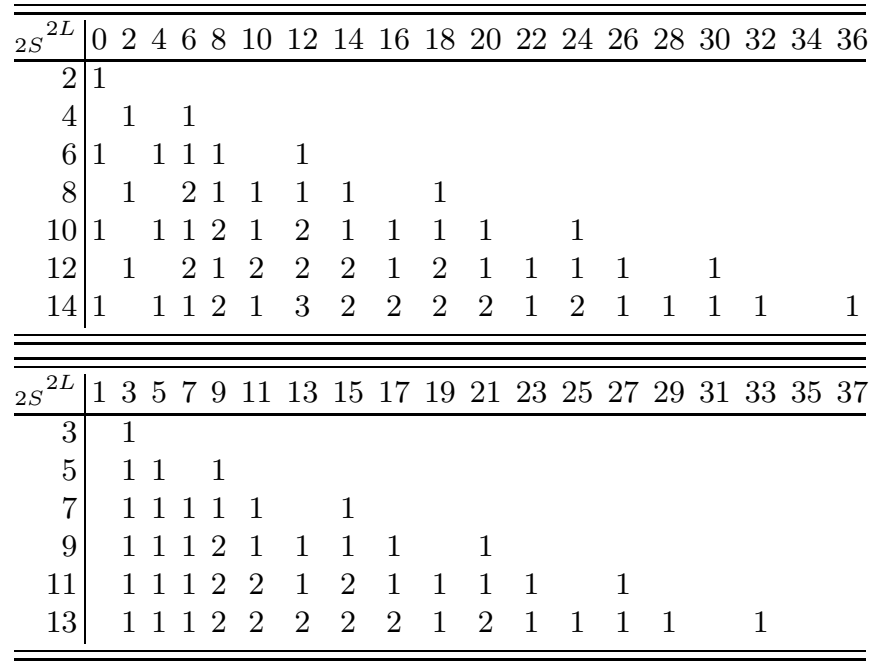

An antisymmetric eigenfunction $\left|l^{3}, L \alpha\right\rangle$ of three electrons each of angular momentum $l$ whose total angular momentum is $L$ can be written as [8]

$$
\left|l^{3}, L \alpha\right\rangle=\sum_{L^{\prime}} F_{L \alpha}\left(L^{\prime}\right)\left|l^{2}, L^{\prime} ; l, L\right\rangle .
$$

Here $\alpha$ is an index which distinguishes different multiplets with the same total angular momentum $L$. $\left|l^{2}, L^{\prime} ; l, L\right\rangle$ is the state in which $l_{1}=l_{2}=l$ are added to obtain pair angular momentum $L^{\prime}$, and then $l_{3}=l$ is added to $L^{\prime}$ to obtain angular momentum $L$. Note that even though $\left|l^{2}, L^{\prime} ; l, L\right\rangle$ is not antisymmetric because $l_{3}$ is treated differently from $l_{1}$ and $l_{2}\left(\left|l^{2}, L^{\prime}\right\rangle\right.$ is antisymmetric under interchange of 1 and 2$),\left|l^{3}, L \alpha\right\rangle$ is antisymmetric. The factor $F_{L \alpha}\left(L^{\prime}\right)$ is called the coefficient of fractional parentage (CFP) associated with pair angular momentum $L^{\prime}$. The two-particle interaction matrix element can be conveniently expressed via the CFP's and the pseudopotential coefficients [6],

$$
\left\langle l^{3}, L \alpha|V| l^{3}, L \beta\right\rangle=3 \sum_{L^{\prime}} F_{L \alpha}\left(L^{\prime}\right) F_{L \beta}\left(L^{\prime}\right) V(R) .
$$

Because of the large Coulomb repulsion at $R=0$, the low lying states will avoid pair angular momentum $L^{\prime}=L^{\prime \text { MAX }}$ (corresponding to $R=0$ ). Where can such states occur? If we choose $L^{\prime}=L^{\prime \mathrm{MAX}}$, then $L$, the total angular momentum, must be greater than or equal to $L^{\prime \mathrm{MAX}}-l$, the minimum possible value of the addition of $L^{\prime \mathrm{MAX}}$ and $l$, the angular momentum of the third electron. Because $L^{\prime \mathrm{MAX}}=2 l-1$, the minimum possible value of $L$ for which $R=0$ is $L=l-1$. States with $L<l-1$ must have $R \geq 2$. Note that although we have selected the pair $\left(l_{1}, l_{2}\right)$ to give $L^{\prime}$, the CFP's

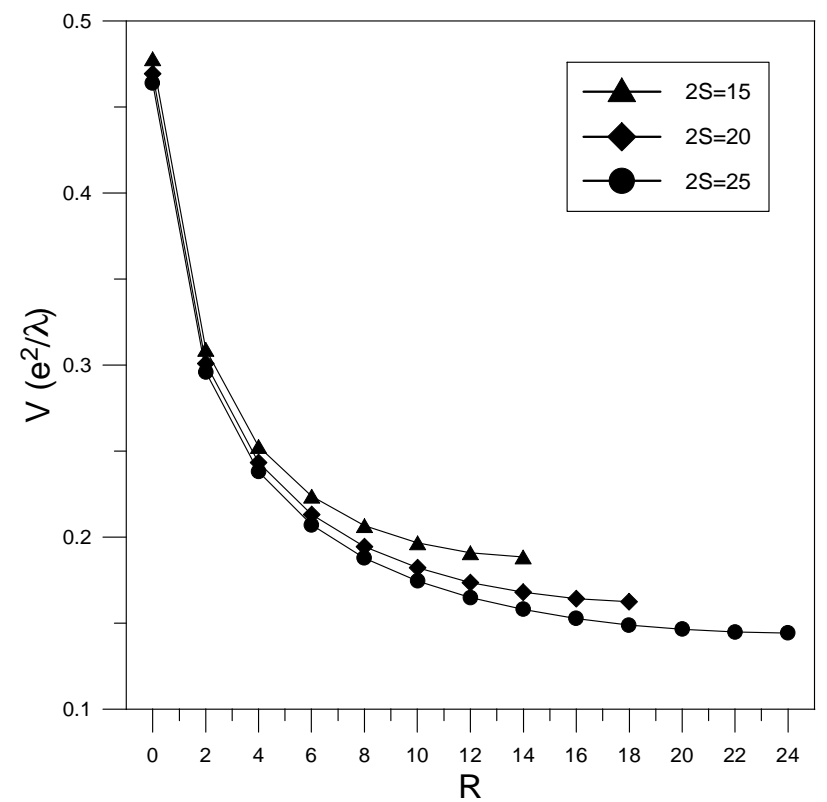

FIG. 1. Plot of $V(R)$, the pseudopotential coefficient of the Coulomb interaction potential, as a function of relative angular momentum $R=L^{\mathrm{MAX}}-L$ of a pair of electrons, for $2 S=15,20$, and 25. $L$ is the pair angular momentum and $L^{\mathrm{MAX}}=2 l-1$ its maximum value. $V(R)$ is measured in units of $e^{2} / \lambda$, where $\lambda$ is the magnetic length

give an eigenfunction of $L$ which is totally antisymmetric. Therefore we need not worry about other pair angular momenta in writing down Eq. (11). The next lower value of $L^{\prime}$ is $L^{\prime \mathrm{MAX}}-2$ (corresponding to $R=2$ ) and states with $L<l-3$ must have $R \geq 4$. Further, states with $L<l-5$ must have $R \geq 6$, and so on. In Tab. II we list the values of $2 L$ for which the CFP with $R=0$ must vanish (i.e. $2 L(R \geq 2)$ ), for which the CFP with $R \leq 2$ must vanish, and for which the CFP with $R \leq 4$ must vanish. The $L=0$ state for $2 S=6$ is the Laughlin $\nu=1 / 3$ state, for $2 S=10$ it is the $\nu=1 / 5$ state, and for $2 S=14$ it is the $\nu=1 / 7$ state. At $2 S=8$ two $L=3$ multiplets occur (see Tab. II). The interparticle interaction must be diagonalized in this two-dimensional subspace. We find that for the linear combination with the lower eigenvalue, the CFP for $R=0$ almost vanishes (its value is less than 0.001). A similar thing occurs at $2 S=9$ for $L=9 / 2$, at $2 S=10$ for $L=4$ and 6 , at $2 S=11$ for $L=9 / 2,11 / 2$, and $15 / 2$, at $2 S=12$ for

TABLE II. The allowed values of $2 L$ for a three electron system that must have $R \geq 2, R \geq 4$, and $R \geq 6$. The listed $2 L$ values are always a subset of the allowed $L$-multiplets given in Tab. I

\begin{tabular}{c|ccccccccl}
\hline \hline $2 l=2 S$ & 6 & 7 & 8 & 9 & 10 & 11 & 12 & 13 & 14 \\
\hline $2 L(R \geq 2)$ & 0 & 3 & 2 & 3,5 & $0,4,6$ & $3,5,7$ & $2,6^{2}, 8$ & $3,5,7,9^{2}$ & $0,4,6,8^{2}, 10$ \\
$2 L(R \geq 4)$ & & & & 0 & 3 & 2 & 3,5 & $0,4,6$ \\
$2 L(R \geq 6)$ & & & & & & & & 0 \\
\hline \hline
\end{tabular}




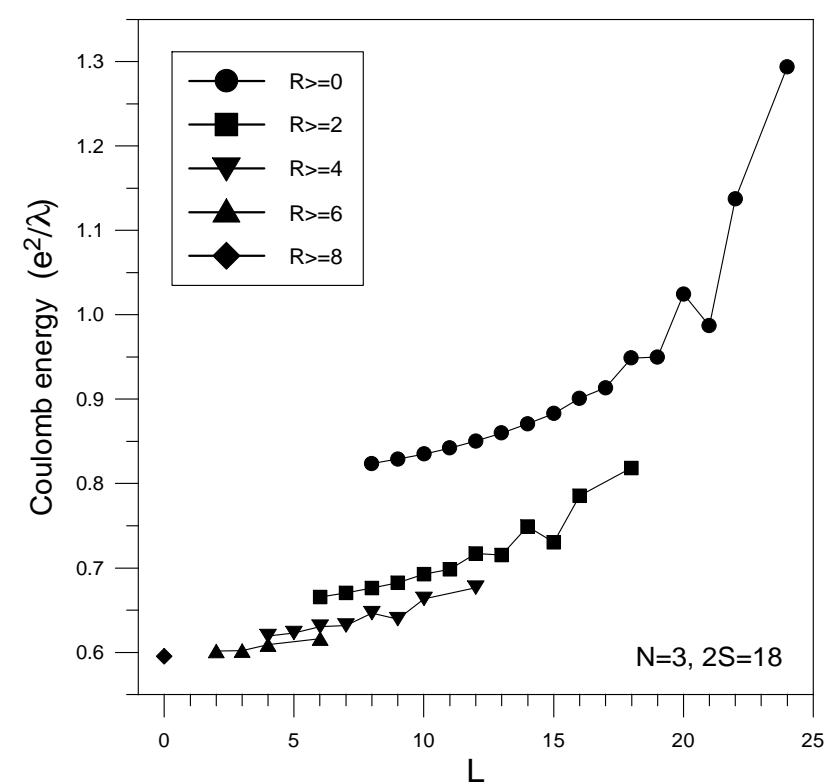

FIG. 2. The Coulomb energy of three electrons with $2 S=18$. The highest energy states have fractional parentage with $R=0$ (dots), next highest with $R=2$ (squares), etc., down to the $L=0$ ground state (diamond) which has no parentage with $R<8$

$L=5,6,7$, and 9 , at $2 S=13$ for $L=11 / 2,13 / 2,15 / 2$, $17 / 2$, and $21 / 2$, and at $2 S=14$ for $L=6^{2}, 7,8,9,10$, and 12 . At $2 S=14$ for $L=6$ there are three allowed multiplets, and diagonalization of the Coulomb interaction gives CFP for $R=0$ which are very small for two of these states. One can see that the subset of states at $2 S-2(N-1)=2 S-4$ of the allowed states at $2 S$ all have CFP for $R=0$ which either vanishes identically or is very small (due to diagonalization of the Coulomb interaction in the subspace of a given $L)$. But $2 S-2(N-1)$ is just $2 S^{*}$ for $2 p_{0}=2$. Thus the $\mathrm{CF}$ picture simply picks the subset of angular momentum multiplets which has $F_{L \alpha}\left(L^{\prime}\right)$ essentially equal to zero for $L^{\prime}=L^{\prime \text { MAX }}$ or $R=0$. For the $\nu=1 / 5$ (or $\nu=1 / 7$ ) state, the effective monopole strength $2 S^{*}=2 S-4(N-1)$ (or $\left.2 S^{*}=2 S-6(N-1)\right)$ picks the subset of states with $R \geq 4$ (or $R \geq 6$ ). The MFCF picture assumes the CFP's for $R<2 p_{0}$ to vanish and simply neglects $V(R)$ for larger $R$.

In Fig. 2 we plot the Coulomb energy as a function of $L$ for the three electron system with $2 S=18$. The dots are $L$-multiplets that have some fractional parentage at $R=0$, the squares have some fractional parentage at $R=2$ but not at $R=0$, etc. Clearly, the gap associated with $V(R=0)$ is the largest, that with $V(R=2)$ the next largest, etc. The $L=0$ ground state (marked by a diamond) corresponds to $\nu=1 / 9$. Its gap is associated with $V(R=8)$ and it is almost unobservable as might be expected. Note that the first excited band in this figure (states with $R \geq 6$ ) contains multiplets at $L=2,3,4,6$, in contrast with the MFCF prediction $(L=1,2,3)$.
Up to here we have concentrated on the three electron system because it is simple and contains the essential physics of our ideas on the validity of MFCF picture. Note that we have not introduced a second energy scale (proportional to $B$ ); the only energy scale is $e^{2} / \lambda(\lambda$ is the magnetic length) and the Coulomb interaction is analyzed in terms of its pseudopotential coefficients $V(R)$. Can we go beyond the three electron system? The answer is yes. The coefficients of fractional grandparentage (CFGP), equivalent to the CFP's in the case of three particles, allow us to write the totally antisymmetric $N$ particle wavefunction as a combination of wavefunctions which are antisymmetric under interchange of particles 1 and 2 and under interchange of particles $3,4, \ldots, N$, and which have well defined angular momentum $L^{\prime}$ of the pair $(1,2)$. The wavefunction and energy expansions are analogous to those given in Eqs. (11) and (2),

$$
\begin{aligned}
& \left|l^{N}, L \alpha\right\rangle=\sum_{L^{\prime}} \sum_{L^{\prime \prime} \alpha^{\prime \prime}} G_{L \alpha, L^{\prime \prime} \alpha^{\prime \prime}}\left(L^{\prime}\right)\left|l^{2}, L^{\prime} ; l^{N-2}, L^{\prime \prime} \alpha^{\prime \prime} ; L\right\rangle, \\
& \left\langle l^{N}, L \alpha|V| l^{N}, L \beta\right\rangle=\frac{N(N-1)}{2} \times \\
& \sum_{L^{\prime}}\left(\sum_{L^{\prime \prime} \alpha^{\prime \prime}} G_{L \alpha, L^{\prime \prime} \alpha^{\prime \prime}}\left(L^{\prime}\right) G_{L \beta, L^{\prime \prime} \alpha^{\prime \prime}}\left(L^{\prime}\right)\right) V(R) .
\end{aligned}
$$

Tables of CFP's and CFGP's are given in nuclear and atomic physics books [8]. If our picture is correct, an $N$ particle system should have bands of states with some fractional parentage for $R \geq 0$, others with $R \geq 2$, etc. In Fig. 3 we show the four electron spectra for $2 S=9$ (Laughlin $\nu=1 / 3$ state) and $2 S=15$ (Laughlin $\nu=1 / 5$ state). In the former case only the low energy $L=0$ state (marked by a square) has $R \geq 2$, all other states have some parentage from $R=0$. In the latter case the entire subset of states which appeared at $2 S=9$ form a low energy band with $R \geq 2$, and the lowest $L=0$ state has $R \geq 4$.

The CFP's and CFGP's can be found in books or computed directly. When a value of $L$ contains a number of multiplets, the set of basis functions and the CFGP's are not uniquely defined in the absence of interaction, which must be diagonalized within the $L$-subspace. We were able to use our numerical code to determine the squares of the CFGP's by diagonalizing the Coulomb interaction, and then by calculating the matrix elements of an interaction where we set $V=1$ for a given $R$ and $V=0$ elsewhere. As follows from Eq. (4), the expectation values obtained in this way determine the squares of CFGP's. In all cases where we expect the CFGP to vanish or to be small compared to the values for neighboring $L$-multiplets, we find that it is so, for $N$ up to 8 and for values of $\nu=1 / 3,2 / 3,2 / 5,2 / 7$, and $2 / 9$.

Two other points are worth mentioning. First, the idea of choosing a subset of the allowed values of $L$ for $N$ Fermions each with angular momentum $l$ by looking 


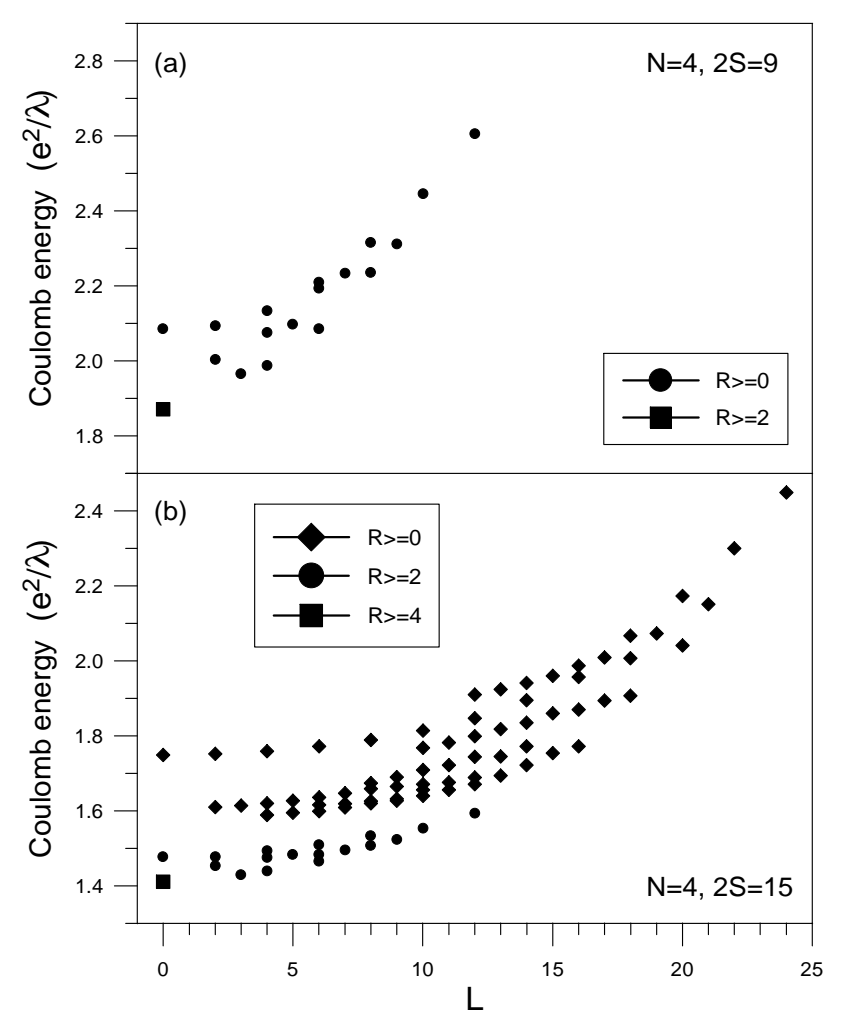

FIG. 3. Energy spectra, $E$ vs. $L$, for a four electron system with $2 S=9$ (a) and $2 S=15$ (b). The states in (a) are a subset of those in (b). In (a) only the lowest state has $R \geq 2$ (square). In (b) the lowest state has $R \geq 4$ (square) and the band of states marked by dots have $R \geq 2$

only at states that can be obtained by adding the angular momentum of $N$ Fermions with $l^{*}=l-p_{0}(N-1)$ is intimately connected with avoiding fractional parentage with $R=0$, or $R=2$ (for $\nu \sim 1 / 5$ ), etc. To have the same concept be valid for residual quasiparticles in a partially filled $\mathrm{CF}$ shell (which would generate $\mathrm{CF}$ hierarchy states [7] like $\nu=4 / 11$ or $4 / 13$ that are outside the Jain sequence), the QP-QP interaction would have to be similar to the Coulomb interaction (i.e. strongly repulsive for $R=0$ and decreasing in magnitude with increasing $R$ ). This does not occur for quasielectrons in the first excited CF shell, but does occur for quasiholes in the lowest $\mathrm{CF}$ shell [7]. Thus it is clear why the $\mathrm{CF}$ hierarchy scheme is often invalid for states outside the Jain sequence. Second, states with a single quasihole (e.g. in the $\nu=1 / 3$ state) have $R$ greater than or equal to the value in the neighboring Laughlin state $(R \geq 2$ for the $\nu=1 / 3$ state), while for a single quasielectron there has to be some parentage for $R$ less than this value (for $R=0$ in the single quasielectron state adjacent to $\nu=1 / 3)$. This explains why the quasielectron energy is larger than the quasihole energy; $V(R=0)$ is much larger than $V(R=2)$.

We have shown that the qualitative results of the MFCF picture can be justified by considering the subset of $L$-multiplets obtained by adding $N$ angular momenta $l^{*}=l-p_{0}(N-1)$. These states have smaller total angular momentum and larger values of the relative angular momentum of pair states. The $L=0$ incompressible ground states at $\nu=\left(1+2 p_{0}\right)^{-1}$ have fractional parentage for values of $R<2 p_{0}$ which is essentially equal to zero, in agreement with the correlation effects proposed in Laughlin's original paper [5]. In fact, for model interactions in which $V(R)$ decreases very rapidly with increasing $R$ (i.e. for very short range interactions), exact diagonalization gives exact zeros of the coefficients of fractional parentage for $R<2 p_{0}$ instead of the very small values obtained with the Coulomb interaction. For fractions containing an integer other than unity in the numerator, we find that the CFGP of one $L=0$ state is small compared to those of other $L$-multiplets, leading to a low-lying incompressible ground state. Only a single energy scale, $e^{2} / \lambda$, the Coulomb scale, is involved in the analysis. The $\mathrm{CF}$ transformation is a convenient way to arrive at a subset of the allowed $L$ values, however the energy scale of the non-interacting composite Fermions is totally irrelevant.

This work was supported in part by the Division of Materials Sciences - Basic Energy Research Program of the U.S. Department of Energy. A. W. acknowledges support from the KBN Grant No. PB674/P03/96/10. The authors would like to thank P.Sitko, K. S. Yi, and D. C. Marinescu for discussions on the preliminary aspects of this work.

* On leave from the Institute of Physics, Wrocław University of Technology, Wrocław, Poland.

[1] J. Jain, Phys. Rev. Lett. 63, 199 (1989).

[2] D. C. Tsui, H. L. Störmer, and A. C. Gossard, Phys. Rev. Lett. 48, 1559 (1982).

[3] F. D. M. Haldane, Phys. Rev. Lett. 51, 605 (1983); F. D. M. Haldane and E. H. Rezayi, Phys. Rev. B 31, 2529 (1985); S. He, X. Xie, and F. Zhang, Phys. Rev. Lett. 68, 3460 (1992); G. Fano, F. Ortolani, and E. Colombo, Phys. Rev. B 34, 2670 (1986).

[4] X. M. Chen and J. J. Quinn, Solid State Commun. 92, 865 (1996).

[5] R. Laughlin, Phys. Rev. Lett. 50, 1395 (1983).

[6] P. Sitko, S. N. Yi, K.-S. Yi, and J. J. Quinn, Phys. Rev. Lett. 76, 3396 (1996).

[7] P. Sitko, K.-S. Yi, and J. J. Quinn, Phys. Rev. B 56, 12417 (1997).

[8] A. de Shalit and I. Talmi, Nuclear Shell Theory, Academic Press, New York and London 1963; M. E. Rose, Elementary Theory of Angular Momentum, John Wiley and Sons, New York, Chapman and Hall, London 1957. 